\title{
Evaluation of a Program to Improve Diabetes Care Through Intensified Care Management Activities and Diabetes Medication Copayment Reduction
}

\author{
Stephen J. Kogut, PhD, MBA; Scott Johnson, PharmD, MS; \\ Tara Higgins, BS Pharm, CDOE; and Brian Quilliam, PhD
}

\begin{abstract}
BACKGROUND: Medication copayment reduction can be integrated with disease management programs to incentivize patient engagement in chronic care management. While disease management programs in diabetes have been evaluated across a range of settings and designs, less is known regarding the effectiveness of copayment reduction as a component of disease management.
\end{abstract}

OBJECTIVE: To evaluate the short-term results of a diabetes-focused disease management program that included copayment reduction, care coordination, and patient goal setting, focusing on rates of evidence-based care processes and all-cause pharmacy and health care costs.

METHODS: Blue Cross \& Blue Shield of Rhode Island offered large employer groups the opportunity to participate in a diabetes disease management initiative that featured reduced copayments (from $\$ 7 / \$ 25 / \$ 40$ for generic, tier 2, and tier 3 drugs, respectively, to $\$ 0$ for generic and $\$ 0-\$ 2$ for brand drugs) for diabetes-related medications. In return for the copayment reduction, participants agreed to the following: (a) participate in care coordination with a case manager, (b) have an annual physical examination, (c) have a hemoglobin A1c blood test at least twice annually, and (d) have a lowdensity lipoprotein cholesterol (LDL-C) test at least once annually. Patients received personalized support provided by a registered nurse and dietician, disease-related education provided by nurses, and intensified case management services, including working with a health coach to establish healthy behavioral change goals. All study subjects were aged 18 years or older and had at least 1 ICD-9-CM code for diabetes and at least 1 claim for an antidiabetic drug during a 12-month measurement period, which was each subject's most recent 12-month period of continuous enrollment from January 1, 2008, through May 31, 2010. Administrative claims data were used to determine the percentage of intervention (participating) and nonintervention (nonparticipating) subjects from among all of the plan's employer groups who received at least once-yearly monitoring of A1c, high-density lipoprotein cholesterol (HDL-C), and LDL-C; medical attention (or drug therapy) for nephropathy; and an eye examination. We conducted multivariate logistic regression analyses to assess the effect of the intervention and other patient characteristics and comorbidities on rates of performance of these care processes, aggregating the 5 processes of care into an "all or none" single composite outcome. We also developed a propensity scoreweighted model to attempt to adjust for differences between the intervention and nonintervention groups resulting from the nonrandomized study design. Additionally, we quantified average plan payments to providers less patient copayments (i.e., net plan cost) per patient per year (PPPY) for the 12-month follow-up period and compared these costs for the intervention versus nonintervention groups.

RESULTS: The study sample consisted of 9,698 patients with diabetes; 649 $(6.7 \%)$ of whom participated in the intervention. $9,049(93.3 \%)$ patients were identified by the insurer as patients with diabetes receiving usual care. Patients in the intervention and nonintervention groups were similarly likely to have all 5 recommended processes of care performed $(40.1 \%$ vs. $38.9 \%$, respectively, $P=0.543)$. Younger patients received all 5 recommended care processes less frequently than older patients $(30.5 \%, 38.0 \%$, and $47.0 \%$ for ages $18-48$ years, $49-59$ years, and 60 years or older, respectively, $P<0.001$ ); in adjusted analyses, patients aged 60 years or older were approximately twice as likely to receive all 5 care processes compared with patients aged 18-48 years (odds ratio [0R] $=1.97,95 \%$ $\mathrm{Cl}=1.75-2.21)$. Users of oral antidiabetic monotherapy were least likely to have these processes of care performed compared with users of multiple oral therapies $(\mathrm{OR}=1.23,95 \% \mathrm{Cl}=1.11-1.36)$ and insulin $(\mathrm{OR}=1.59,95 \%$ $\mathrm{Cl}=1.41-1.78$ ). PPPY prescription drug costs incurred by the plan were greater for intervention than comparison patients (means [SDs] of $\$ 3,139$ $[\$ 3,426]$ vs. $\$ 2,854$ [ $\$ 3,938]$, respectively, $P<0.001)$; and the genericdispensing ratio was slightly lower (means [SDs] of $62.1 \%$ [22.4\%] and $65.4 \%$ [23.0\%], respectively, $P<0.001$ ). There were no significant differences between the intervention and comparison groups in mean [SD] PPPY all-cause medical care costs $(\$ 7,475$ [\$17,601] vs. \$8,577 [\$22,972], respectively, $P=0.213)$ or total all-cause costs $(\$ 10,613$ [\$18,590] vs. $\$ 11,431$ [\$24,060], $P=0.666$ ).

CONCLUSIONS: Patients participating in this incentive program featuring diabetes medication copayment reduction and disease management components did not receive recommended care any more or less frequently than other enrolled members with diabetes. Younger patients and those utilizing oral antidiabetic monotherapy as their drug regimens were less likely to have the recommended processes of care performed. While prescription drug expenditures incurred by the plan were greater for intervention patients, between-group differences in total costs for medications and all-cause medical care were not statistically significant. Further follow-up is required to determine the success of this program over the longer term in promoting quality of care and achieving cost reductions and improved health outcomes.

J Manag Care Pharm. 2012;18(4):297-310

Copyright $\odot 2012$, Academy of Managed Care Pharmacy. All rights reserved. 


\section{Evaluation of a Program to Improve Diabetes Care Through Intensified Care Management Activities and Diabetes Medication Copayment Reduction}

\section{What is already known about this subject}

- Although diabetes management has improved during the past decade, there is opportunity for improvement. The National Committee on Quality Assurance (NCQA) Healthcare Effectiveness Data and Information Set (2009) indicated that approximately $89 \%$ of commercially insured patients with diabetes had hemoglobin Alc measured at least once yearly, while rates for low-density lipoprotein cholesterol (LDL-C) screening and monitoring for nephropathy were $85 \%$ and $82 \%$, respectively. Lower rates were reported for the performance of diabetic eye examinations (57\%). The 2010 National Healthcare Quality Report found that in $2007,88 \%$ of patients with diabetes had Alc measured, and $61 \%$ received eye examinations.

- The Medicare Health Support Pilot Program (2011), which randomized approximately 240,000 Medicare beneficiaries with diabetes or heart failure to either standard care or a disease management intervention employing remote call centers staffed by nurses, found no significant between-group differences in hospital admissions or overall costs of care.

- A cluster-randomized trial by Sönnichsen et al. (2010) evaluated a disease management intervention comprising interdisciplinary care, face-to-face physician and patient education, standardized medication documentation, and shared physician-patient goal setting. Compared with 840 study patients receiving usual care, the 649 intervention patients more frequently had recommended care performed (e.g., eye and foot examinations and Alc measurement) and experienced reductions in body mass index and blood cholesterol. However, statistical analyses revealed no sustained between-group differences in Alc reduction over time.

- Less is known about the outcomes of copayment reductions in disease management programs. Gibson et al. (2011) described the outcomes of copayment reduction for diabetes medications, separately and when coupled with a disease management program. Among disease management participants, but not among nonparticipants, drug copayment reductions were associated with higher rates of completion of Alc, lipid testing, and urinalysis, and results were sustained over a 3-year follow-up period. In a study of an initiative implemented by one large employer, Chernew et al. (2008) found that copayment reductions from $\$ 5 / \$ 25 / \$ 45$ to $\$ 0 / \$ 12.50 / \$ 22.50$ for generic, tier 2 , and tier 3 medications, respectively, were associated with a 4.02 percentage point increase in medication possession ratio $(P<0.001)$.

\section{What this study adds}

- Patients volunteering to participate in a diabetes-focused disease management and copayment incentive program had generally high rates of annual performance of 4 important elements of diabetes care that were similar to those of the plan's patients with diabetes who did not participate in the program (high-density lipoprotein cholesterol test: $85.7 \%$ vs. $89.7 \%, P=0.001$; LDL-C

\section{What this study adds (continued)}

test: $86.7 \%$ vs. $89.8 \%, P=0.014$; Alc test: $92.3 \%$ vs. $94.1 \%$, $P=0.064$; medical attention for nephropathy: $83.8 \%$ vs. $83.3 \%$, $P=0.726$, respectively). Rates were lower overall for eye examinations, yet similar among intervention versus nonintervention patients $(51.2 \%$ vs. $48.0 \%, P=0.114)$

- The average 12-month per member all-cause plan cost for medications and medical care among intervention patients was similar to cost for members with diabetes who did not participate in the program ( $\$ 10,613$ vs. $\$ 11,430$, respectively, $P=0.666)$. Average all-cause medication cost was higher for intervention than comparison group patients ( $\$ 3,139$ vs. $\$ 2,854$, respectively, $P<0.001$ ) and the average generic-dispensing ratio was lower $(62.1 \%$ vs. $65.4 \%$, respectively, $P<0.001$ )

- The odds of receiving all 5 recommended care processes were twice as high for patients aged 60 years or older compared with patients aged 18 to 48 years (odds ratio $[\mathrm{OR}]=1.97,95 \% \mathrm{CI}=1.75$ 2.21). Compared with users of oral monotherapy, odds were $23 \%$ higher for users of multiple oral therapies (OR $=1.23,95 \%$ $\mathrm{CI}=1.11-1.36)$ and $59 \%$ higher for insulin users $(\mathrm{OR}=1.59,95 \%$ $\mathrm{CI}=1.41-1.78)$.

$\mathrm{D}$ iabetes mellitus is a major burden on the U.S. health care system. Estimates from the U.S. Centers for Disease Control indicate that approximately 26 million people in the United States are living with diabetes, including 7 million Americans who are undiagnosed, while nearly 2 million adults were newly diagnosed with diabetes during 2010. ${ }^{1}$ It is estimated that 1 in 3 people in the United States will develop diabetes during their lifetime. ${ }^{2}$ Diabetes is the sixth leading cause of death in the United States, contributing to approximately 225,000 deaths yearly. ${ }^{3}$ In 2002, diabetes contributed to 16.9 million days of hospitalization and 62.6 million physician office visits, and total average health care expenditures were $\$ 13,243$ for every person with diabetes compared with $\$ 5,642$ for every person without diabetes, controlling for age and other demographic characteristics. ${ }^{4}$ These figures underscore why the health care system continues to devise and implement interventions to manage the disease and reduce diabetesrelated complications and associated costs.

As a result of the high prevalence ${ }^{5}$ and cost burden ${ }^{6}$ associated with diabetes and its complications, managed care organizations are directing significant resources towards ensuring that evidence-based care is routinely delivered to promote risk reduction and avert untoward health outcomes. Quality of care recommendations for diabetes supported by the American Diabetes Association include the routine measurement of glycosylated hemoglobin (Alc), blood lipids and renal function, and routine diabetic retinal examinations for preventing disease complications. ${ }^{7}$ Poor glycemic control and dyslipidemia are significant risk factors for coronary artery disease (CAD) 


\section{Evaluation of a Program to Improve Diabetes Care Through Intensified Care Management Activities and Diabetes Medication Copayment Reduction}

in patients with diabetes. For example, using data from the United Kingdom Prospective Diabetes Study to assess risk factors for CAD, Turner et al. (1998) reported a hazard ratio (HR) of 2.26 for low-density lipoprotein cholesterol (LDL-C; 95\% confidence interval $[\mathrm{CI}]=1.70-3.00)$; $\mathrm{HR}=1.52$ for Alc $(95 \%$ $\mathrm{CI}=1.15-2.01)$; and $\mathrm{HR}=0.55$ for high-density lipoprotein cholesterol (HDL-C; 95\% CI=0.41-0.73), comparing patients categorized within the upper-third versus lowest-third levels. ${ }^{8}$ The prevention of nephropathy and retinopathy is an additionally important area of focus. According to data from the National Health and Nutrition Examination Survey (NHANES IV), approximately $40 \%$ of patients with type 2 diabetes have some degree of chronic kidney disease, findings that underscore the importance of early detection and intervention. ${ }^{9}$ Zhang et al. (2010) determined the prevalence of retinopathy among NHANES IV participants with diabetes receiving a medical examination, finding that $4.4 \%$ had retinopathy classified as being a threat to vision. ${ }^{10}$

Results from the 2009 National Committee on Quality Assurance (NCQA) Healthcare Effectiveness Data and Information Set (HEDIS) reveal a generally high level of performance of diabetes-related quality of care processes among patients enrolled in commercial plans: approximately $89 \%$ of patients with diabetes had their Alc measured at least once yearly, while rates for LDL-C screening and monitoring for nephropathy were $85 \%$ and $82 \%$, respectively. ${ }^{11}$ Lower rates were reported for the performance of diabetic eye examinations (57\%). These results are similar to the findings reported in the 2010 National Healthcare Quality Report, which revealed that $88 \%$ of patients with diabetes had their Alc measured in 2007 , while eye examinations were received by $61 \%$ of patients. ${ }^{12}$ These results also indicate opportunity for improving these core measures of routine diabetes care.

Disease management approaches in diabetes have included components such as enhanced patient education, improved care coordination, increased involvement of pharmacists, and the use of remote call centers for patient support. The Medicare Health Support (MHS) Pilot Program, which is the largest evaluation of disease management delivered to older patients with diabetes published to date, found that disease management programs employing remote call centers staffed by nurses were ineffective in decreasing hospital admissions or reducing overall costs of care. ${ }^{13}$ In the MHS, more than 240,000 patients with diabetes or heart failure were randomized to either the disease management intervention or standard care. Improvements were noted for only 14 of 40 of the processes of care measured, with only small percentage point changes observed.

A cluster-randomized trial conducted by Sönnichsen et al. (2010) evaluated a disease management intervention comprising interdisciplinary care, face-to-face physician and patient education, standardized medication documentation, and shared physician-patient goal setting delivered to 649 Austrian patients with diabetes. ${ }^{14}$ Compared with the 840 study patients receiving usual care, intervention patients more frequently had recommended care performed (e.g., eye and foot examinations and Alc measurement) and experienced reductions in body mass index and blood cholesterol. A small improvement (0.13 percentage point) was observed in reduction of Alc among intervention patients, yet the difference in Alc reduction between intervention and control patients was not sustained after statistical adjustment for cluster effects and status at baseline. ${ }^{14}$ A randomized trial by Hogg et al. (2009) found that Anticipatory and Preventive Team Care (APTCare) involving nurse practitioners and pharmacists delivered to 120 patients yielded improvements in guideline-based processes of care across several conditions including diabetes. ${ }^{15}$ However, the improvements in the diabetes-specific measures were not statistically significant nor were significant differences observed in rates of hospitalization or patient quality of life between the intervention and usual care groups.

Disease management programs including diabetes medication copayment reduction or waiver have been evaluated as a means of improving diabetes care and reducing cost. Noteworthy models include the Asheville Diabetes Care Project (Asheville Project) ${ }^{16}$ and the subsequent Diabetes Ten City Challenge. ${ }^{17}$ While the primary focus of the Asheville Project was to apply pharmaceutical care services specific to diabetes management, the initiative also offered participants copayment waivers for diabetes medications and supplies. The evaluation measures employed in the Asheville Project focused upon the achievement of therapeutic goals; patients who did not have Alc and lipid measurements performed were considered lost to follow-up. Participants in the Asheville Project reported that the medication copayment waiver was a highly important factor in agreeing to participate in the program. ${ }^{18}$ Results of the Diabetes Ten City Challenge included increases in rates of performance of several diabetes-related processes of care, including eye examinations (from $57 \%$ at baseline to $81 \%$ ), yearly Alc testing (54\% to 97\%), and yearly lipid profile tests (51\% to $92 \%$ ), while average total health care costs per person were reduced by $\$ 1,080$ for the 1-year study period. ${ }^{17}$

However, the improvements in diabetes care and outcomes reported in these 2 studies should be interpreted cautiously in consideration of the nonexperimental designs employed and the health status of study participants at baseline. Patients participating in the Asheville Project were not randomized to the intervention, and a majority (more than 61\%) of patients entered the study with poorly controlled diabetes (Alc level exceeding 7\%). In the Diabetes Ten City Challenge, patient enrollment was voluntary, and the study employed a pre-post design without a control group comparison. While improvements in rates of performance of diabetes-related care processes were substantial, it is uncertain if these improvements differed from usual care patterns among the employers' nonparticipating employees with diabetes. 


\section{Evaluation of a Program to Improve Diabetes Care Through Intensified Care Management Activities and Diabetes Medication Copayment Reduction}

In the years following these 2 important studies, the value of medication therapies has been a growing consideration in formulary designs, ${ }^{19,20}$ and the results of comparative effectiveness research may yet support copayment variation based on expected value for a particular patient's clinical circumstance. ${ }^{21}$ Yet, there has been a paucity of research examining the effect of copayment waiver or reduction on patient health outcomes, quality of care, and cost.

Gibson et al. (2011) described the outcomes of a value-based insurance design including copayment reduction for diabetes medications, as evaluated separately and when coupled with a disease management program. ${ }^{22}$ This was a voluntary program offered by one large employer to all of its employees with diabetes. These researchers found that the intervention including both the pharmacy copayment reduction and disease management components yielded higher rates of completion of Alc, lipid testing, and urinalysis, compared with disease management alone. The results were sustained over a 3-year follow-up period. Chernew et al. (2008) $)^{23}$ and Chang et al. (2010) ${ }^{24}$ also reported improvements in diabetes medication adherence associated with copayment reduction or waiver, although neither of these studies employed a randomized design, and in the study by Chang et al., copayment reduction was not incorporated within a defined disease management program.

In 2008, Blue Cross \& Blue Shield of Rhode Island (RI) initiated an employer-based voluntary pilot program to improve diabetes-related care. Our aim was to describe the association of the program with the quality of care received, focusing upon the performance of 5 diabetes-related processes of care: annual LDL-C, HDL-C, and Alc testing; medical attention for nephropathy; and eye examinations. We sought to determine if patients participating in the program more frequently received these processes of care during a 12-month period compared with all other Blue Cross \& Blue Shield of RI members with diabetes who were not enrolled in the program. Between these groups, we also compared the average per-member costs associated with the utilization of prescription drugs and medical services.

\section{Methods}

\section{Design and Intervention}

We employed a retrospective, cross-sectional design, using electronic claims data to describe patients' health service utilization and associated cost during a 12-month time frame between January 1, 2008, and May 31, 2010. All patients had at least 12 continuous months of enrollment and were at least 18 years of age. The data sources used in this analysis included medical, pharmacy, and enrollment data files, providing demographic information, medical diagnoses, health care procedures, medication dispensings, and hospital discharge records. These data included associated costs where applicable.

The main elements of the program included disease education, counseling, and close oversight provided by assigned nurse case managers and copayment reductions for diabetes-related medications. Specifically, medication copayments for intervention members were reduced to either $\$ 2.00$ for brand name medications and $\$ 0$ (zero) for generic medications, or to a $\$ 0$ copayment for both brand and generic antidiabetic medications, depending upon the account. The 3-tier pharmacy benefit design for members not participating in the program remained as $\$ 7 / \$ 25 / \$ 40$ for generic, tier 2 , and tier 3 medications, respectively. The program required that participants agree to the following: (a) participate in care coordination with their case managers, (b) have an annual physical exam, (c) have an Alc blood test at least twice a year, and (d) have an LDL-C test performed at least once per year. Additionally, patients received personalized support provided by a registered nurse and dietician, which included working to achieve healthrelated goals. Patients also received disease-related education provided by nurses and intensified case management services, including working with a health coach to establish healthy behavioral change goals. Members also completed a contract that pledged their agreement to remain engaged in care and to receive recommended tests and health exams. Participants were informed that the copayment reduction would be rescinded if they did not receive recommended tests and participate in care coordination. Patients not participating in the program received usual care, which included the plan's usual disease management components such as the provision of educational materials and case management following a hospital visit, but without personalized goal setting or copayment reductions.

The diabetes care management activities and diabetes medication copayment reduction program (i.e., the "intervention") was offered to the plan's larger employer groups with 1,000 or more employees. Five of these groups agreed to participate. Patient recruitment was performed by Blue Cross \& Blue Shield of RI, which had sole responsibility for identifying members with diabetes in the participating employer groups; invitations to participate in the program were mailed by the health plan to these patients' homes. The mailings were followed by a telephone voice reminder message sent 2 weeks later.

The intervention group for the study comprised those members who were offered the program and agreed to participate through May 31, 2010. For these patients, the study time frame consisted of the most recent 12 months of participation in the program. The comparison group comprised all other members identified by the plan as having diabetes during the time frame, including those members from both the large employer groups from which the intervention patients were recruited and from among all of the plan's other groups. For the comparison cohort, the most recent 12 months of continuous enrollment comprised the study time frame. All patients in both groups received a dispensing for either an oral or injectable medication for diabetes during the 12-month study time 


\section{Evaluation of a Program to Improve Diabetes Care Through Intensified Care Management Activities and Diabetes Medication Copayment Reduction}

\section{FIGURE 1 Study Inclusion and Exclusion Criteria}

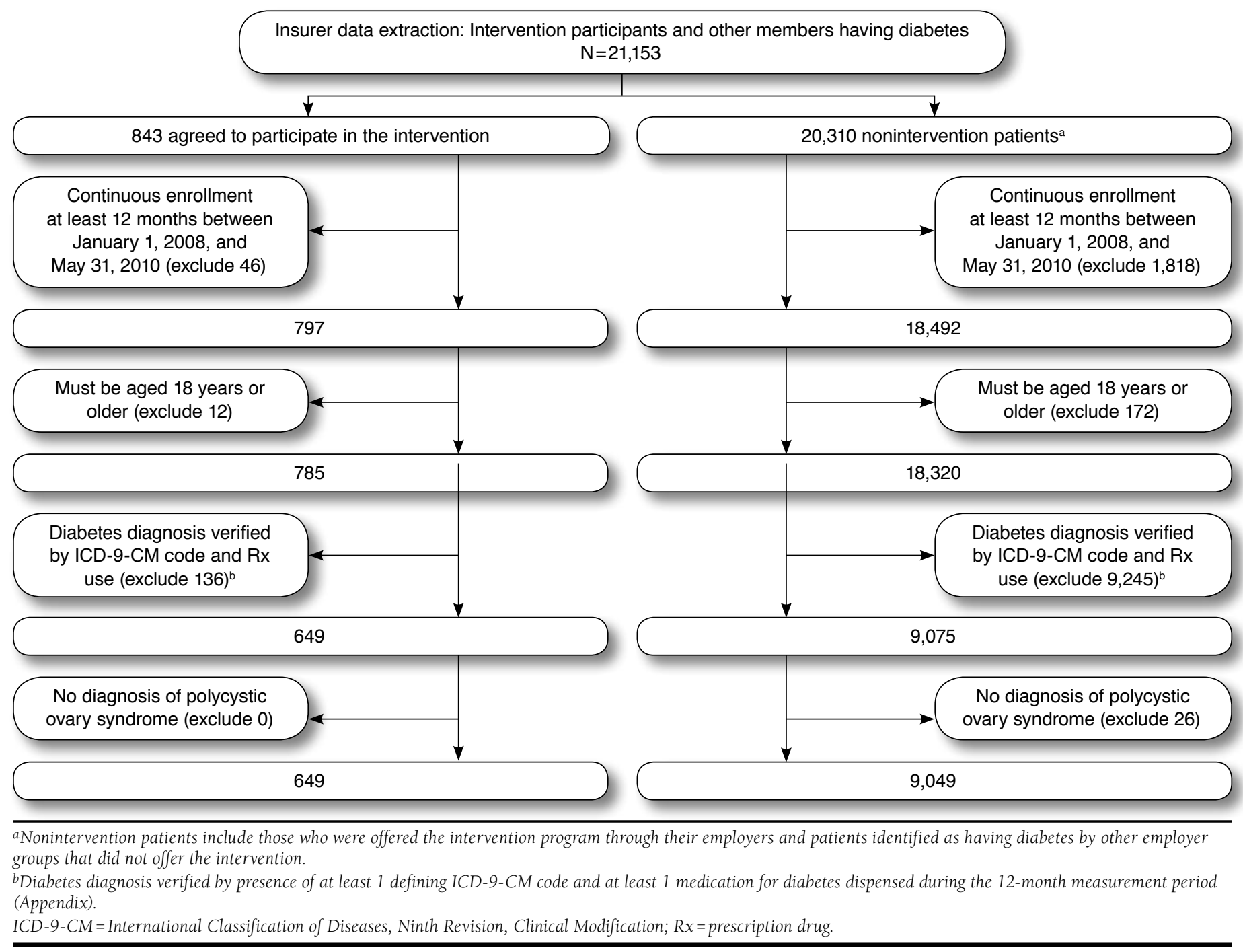

frame and had a diagnosis of diabetes documented during this 12-month period. We excluded patients who had a primary or secondary diagnosis of polycystic ovary syndrome, for which metformin may be prescribed. The flowchart in Figure 1 presents an overview of the study inclusion criteria and sequence.

\section{Study Outcomes and Statistical Analyses}

Our main objectives were to compare (a) rates of performance of recommended diabetes-related care processes between the intervention and nonintervention groups and (b) allcause pharmacy and medical care costs between groups. We determined the percentages of patients who received at least once-yearly monitoring of Alc, HDL-C, LDL-C, and medical attention for nephropathy, which included evidence of renal function monitoring, a diagnosis of nephropathy, or use of an angiotensin-converting enzyme inhibitor or angiotensin II receptor blocker (at least 1 pharmacy claim during the measurement period). We also determined if patients received a dilated eye exam during this time span.

We created an "all or none" single composite outcome comprising all these aspects of recommended care. Our measurement specifications were based on the 2009 HEDIS diagnosis and procedure code listings, ${ }^{25}$ with the exception of eye examinations; due to our 12-month measurement time frame we were unable give credit for negative retinal exam results obtained in the prior year, as the HEDIS methodology allows. Annual HDL-C monitoring is not included in the HEDIS comprehensive diabetes care measure set. Our measure was 


\section{Evaluation of a Program to Improve Diabetes Care Through Intensified Care Management Activities and Diabetes Medication Copayment Reduction}

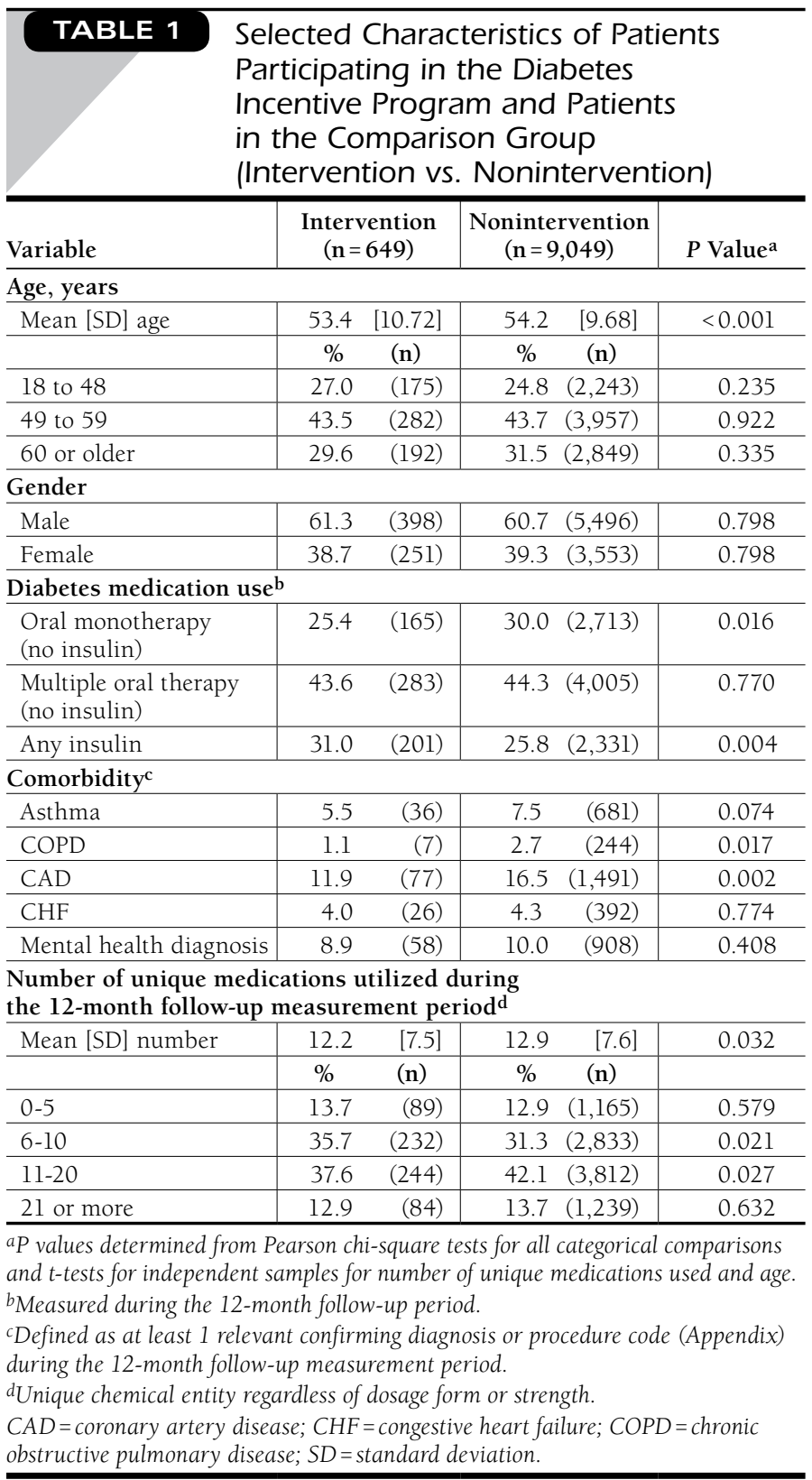

calculated using the Current Procedural Terminology (CPT) codes for lipid panel and HDL-C testing as acceptable numerator qualifiers. The Appendix presents a listing of International Classification of Diseases, Ninth Revision, Clinical Modification (ICD-9-CM) and CPT codes used in identifying diagnoses and processes of care.

In addition to comparing rates of these processes of care between groups, the intervention and nonintervention groups were compared according to age, gender, type of diabetes medication used, and comorbidities. Most of the patients enrolled by this commercial insurer were middle-aged, nonelderly working adults and their family members. We created age categories that roughly approximated tertiles of the distribution, while also maintaining at least a 10-year range for the middle strata. Medication burden was determined according to the number of unique medications utilized during the 12-month study time period, identified via National Drug Code (NDC) numbers. This variable represented the sum of unique medications utilized during the 12-month study period, counting each chemical entity only once, regardless of the number of dispensings or the dosage form or strength. The type of diabetes medication regimen used during the 12month period was classified as follows: (a) oral monotherapy without insulin use; (b) multiple oral therapy without insulin use; or (c) any insulin use, with or without concomitant oral medication use, also identified using product NDC numbers. We also identified the following comorbidities via relevant ICD-9-CM codes (appearing at least once during the 12-month time frame): asthma, chronic obstructive pulmonary disease (COPD), CAD, heart failure, and a mental health condition, which included the diagnoses of depression, bipolar disorder, and schizophrenia.

Rates of performance were determined for each of the diabetes-related processes of care and for the aggregated composite measure, and these rates were stratified by group status and according to the covariates identified above. Pearson chisquare tests were applied to determine the statistical significance of differences in these rates and also to assess differences in patient characteristics and the presence of comorbidities between the intervention and nonintervention groups.

A multivariate logistic model was developed to determine the effect of the intervention on the outcome of the "all or none" measure, defined as having all of the measured processes of care performed during the 12-month period. The model was created using a manual backward stepwise process. The log-likelihood test was used to assess the multivariate model at each step, removing least statistically significant covariates with each iteration and evaluating differences between full and reduced models for statistical significance $(P<0.05)$. Gender and age remained in the model throughout. The HosmerLemeshow goodness-of-fit test was used to assess the calibration of the final model. The measure of effect was presented as an odds ratio (OR) with corresponding 95\% CIs.

To mitigate bias resulting from the nonrandomized design, an inverse propensity score-weighted model was also utilized to adjust for the likelihood of intervention group inclusion. A 2-stage propensity score approach was applied as described by D'Agostino (1998), ${ }^{26}$ which attempted to account for differences in diabetes severity and comorbidities between groups, recognizing that volunteers for the diabetes management program may have differed in health status from the plan members with diabetes who did not participate in the intervention. The first 


\section{Evaluation of a Program to Improve Diabetes Care Through Intensified Care Management Activities and Diabetes Medication Copayment Reduction}

\section{TABLE 2 Frequency and Percentage of Patients with Diabetes Receiving}

Recommended Care by Group Status and Other Patient Characteristics ${ }^{a}$

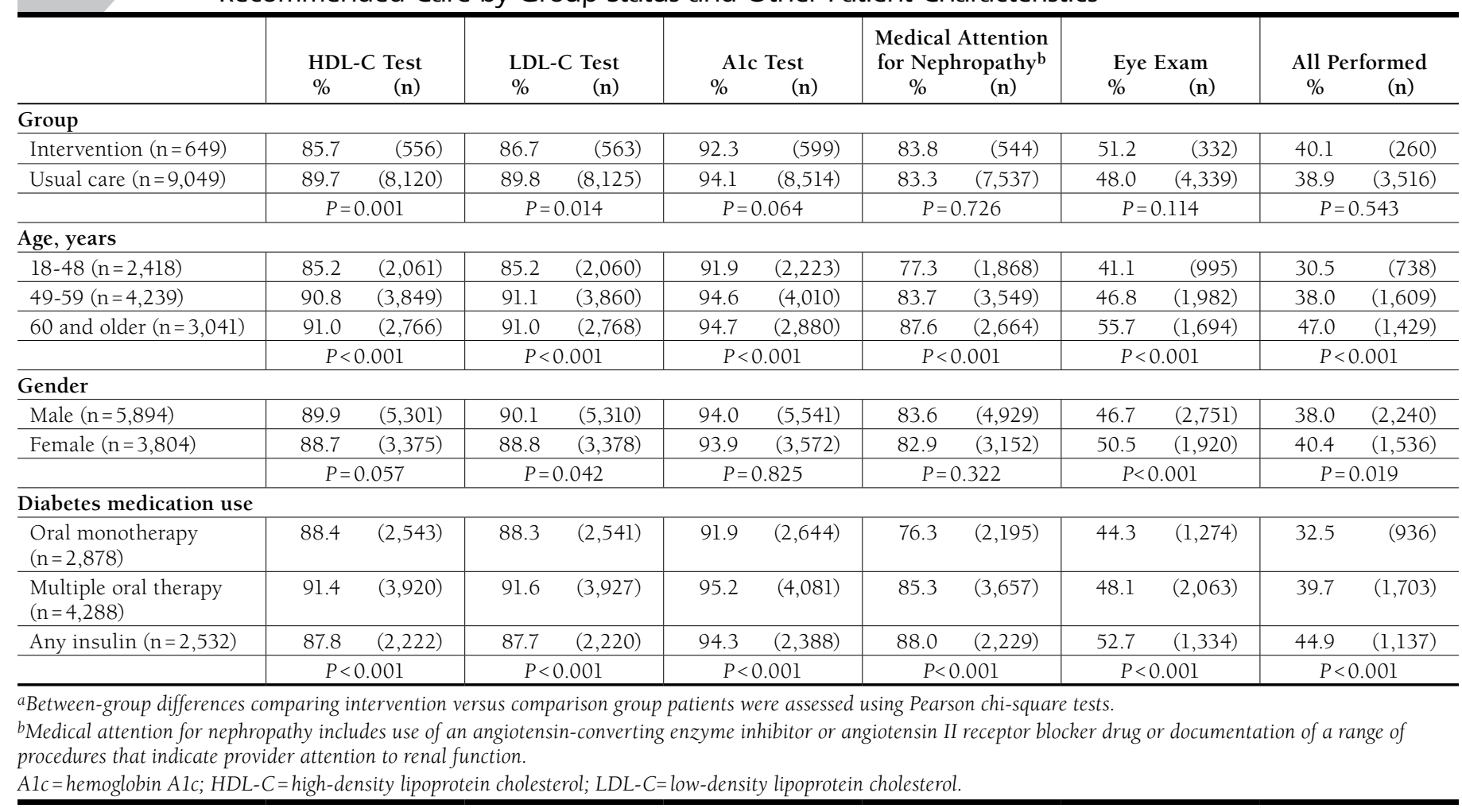

stage of this approach aims to predict treatment group (i.e., intervention vs. nonintervention status) according to available covariates. Variables assessed for incorporation into the propensity score included the type of diabetes medication utilized, the comorbidities identified above, and the number of unique medications utilized during the study time frame. Gender and age remained in the model throughout, while other variables were included if they were significant $(P<0.05)$ in building a fitted multiple logistic regression model having an outcome variable representing intervention group status. A correlation matrix was used to identify the presence of multicollinearity, and potential interactions between variables were explored. In the second stage, the inverse propensity-score weight was incorporated in a model predicting the diabetes processes of care composite measure and including intervention group status as a weight-adjusted predictor.

Additionally, we calculated the 12-month costs of all-cause medical care, pharmacy, and total health expenditures and compared these costs for the intervention versus nonintervention groups. The Wilcoxon rank-sum (Mann-Whitney U) test was used to evaluate differences between cost values. For all analyses, we assumed an a priori significance level of $P<0.05$ (2-sided). Data analyses were performed using SAS version 9.2 (SAS Institute Inc., Cary, NC).

\section{Results}

The insurer identified 21,153 patients with diabetes, 843 of whom agreed to participate in the diabetes incentive program; 20,310 were members identified as having diabetes using algorithms employed by the plan but did not participate in the program. Approximately 5.5\% of intervention patients and $9.0 \%$ of nonintervention patients were excluded after application of the continuous enrollment criterion. Among those enrolled for at least 12 months, 12 intervention patients and 172 nonintervention patients were less than 18 years of age and were excluded. We required that patients had at least 1 diagnosis of diabetes and at least 1 dispensing of an antidiabetic medication during the 12-month period. This criterion resulted in the additional exclusion of 136 intervention and 9,245 nonintervention patients. After excluding 26 patients from the nonintervention group with a diagnosis of polycystic ovary disease, the final study sample consisted of 9,698 patients with diabetes, 649 of $(6.7 \%)$ of whom were participants in the intervention. No intervention patients withdrew from the program during the study time frame.

The mean (standard deviation [SD]) ages of patients in the intervention and nonintervention groups were similar (53 [10.7] vs. 54 [9.7] years, respectively), as were the percentages 


\section{Evaluation of a Program to Improve Diabetes Care Through Intensified Care Management Activities and Diabetes Medication Copayment Reduction}

\section{TABLE 3 Multivariate Logistic Regression Model: ${ }^{\text {Patient Covariates and }}$ Intervention Group Status as \\ Predictors of Receiving All Diabetes- Related Processes of Care}

\begin{tabular}{|c|c|c|c|}
\hline Characteristic (n) & $\begin{array}{c}\text { Beta } \\
\text { Coefficient }\end{array}$ & $\begin{array}{l}\text { Standard } \\
\text { Error }\end{array}$ & $\begin{array}{l}\text { Odds Ratio } \\
(95 \% \mathrm{CI})\end{array}$ \\
\hline \multicolumn{4}{|l|}{ Group } \\
\hline Usual care $(9,049)$ & - & - & Reference \\
\hline Intervention (649) & 0.0588 & 0.0847 & $1.061(0.898-1.252)$ \\
\hline \multicolumn{4}{|l|}{ Age, years } \\
\hline $18-48(2,418)$ & - & - & Reference \\
\hline $49-59(4,239)$ & 0.3181 & 0.0557 & $1.375(1.233-1.533)$ \\
\hline 60 and older $(3,041)$ & 0.6759 & 0.0602 & $1.966(1.747-2.212)$ \\
\hline \multicolumn{4}{|l|}{ Gender } \\
\hline Female $(3,804)$ & - & - & Reference \\
\hline Male $(5,894)$ & -0.0678 & 0.0446 & $0.934(0.856-1.020)$ \\
\hline \multicolumn{4}{|c|}{ Diabetes medication use } \\
\hline $\begin{array}{l}\text { Oral monotherapy } \\
(2,878)\end{array}$ & - & - & Reference \\
\hline $\begin{array}{l}\text { Multiple oral therapy } \\
(4,288)\end{array}$ & 0.2064 & 0.0525 & $1.229(1.109-1.363)$ \\
\hline Any insulin $(2,532)$ & 0.4621 & 0.0592 & $1.587(1.414-1.783)$ \\
\hline \multicolumn{4}{|l|}{ Comorbidity } \\
\hline Reference $^{b}$ & - & - & 1.0 \\
\hline Asthma (717) & -0.1176 & 0.0836 & $0.889(0.755-1.047)$ \\
\hline CAD $(1,568)$ & 0.0393 & 0.0620 & $1.040(0.921-1.174)$ \\
\hline CHF (418) & 0.0178 & 0.1086 & $0.982(0.794-1.216)$ \\
\hline COPD (251) & -0.2410 & 0.1366 & $0.786(0.601-1.027)$ \\
\hline $\begin{array}{l}\text { Mental health } \\
\text { diagnosis }(966)\end{array}$ & -0.0818 & 0.0730 & $0.921(0.799-1.063)$ \\
\hline \multicolumn{4}{|c|}{$\begin{array}{l}\text { Number of unique medications utilized } \\
\text { during the 12-month follow-up period }\end{array}$} \\
\hline $0-5(1,254)$ & - & - & Reference \\
\hline $6-10(3,065)$ & 0.3229 & 0.0759 & $1.381(1.190-1.603)$ \\
\hline $11-20(4,056)$ & 0.6217 & 0.0750 & $1.862(1.607-2.157)$ \\
\hline 21 or more $(1,323)$ & 0.7000 & 0.0930 & $2.014(1.678-2.417)$ \\
\hline \multicolumn{4}{|c|}{$\begin{array}{l}{ }^{a} C \text {-statistic }=0.60 . \\
{ }^{b} \text { Reference group is the absence of the particular comorbidity. } \\
C A D=\text { coronary artery disease } C H F=\text { congestive heart failure; } C I=\text { confidence } \\
\text { interval; } C O P D=\text { chronic obstructive pulmonary disease. }\end{array}$} \\
\hline
\end{tabular}

of patients within each age stratum among these groups (Table 1). Males represented $61 \%$ of both the intervention and nonintervention groups. A higher percentage of patients in the intervention group were insulin users ( $31.0 \%$ vs. $25.8 \%, P=0.004$ ), while in the nonintervention group a higher percentage of patients were users of oral antidiabetic monotherapy (30.0\% vs. $25.4 \%, P=0.016)$. Comorbidities, identified by relevant diagnosis codes documented during the 12-month period, were more frequent among nonintervention patients than intervention patients, with statistically significant differences observed for the prevalence of COPD (2.7\% vs. $1.1 \%$, respectively, $P=0.017)$ and $\operatorname{CAD}(16.5 \%$ vs. $11.9 \%, P=0.002)$. The mean number of unique medications utilized per patient during the 12-month period was 12.2 for the intervention group compared with 12.9 for the nonintervention group $(P=0.032)$.

Patients in the intervention group were as likely as nonintervention patients to have all of the recommended processes of care performed $(40.1 \%$ vs. $38.9 \%$, respectively, $P=0.543$; Table 2). Among all processes of care, annual Alc testing was performed with the greatest frequency $(92.3 \%$ of patients in the intervention group; $94.1 \%$ of nonparticipating patients, $P=0.064$ ). Patients in the intervention group had lipid monitoring tests performed slightly less frequently than nonintervention patients (HDL-C testing: intervention group $85.7 \%$ vs. nonintervention group $89.7 \%, P=0.001$; LDL-C testing: intervention group $86.7 \%$ vs. nonintervention group $89.8 \%$, $P=0.014$ ). Rates of renal function testing (or use of a renalprotective medication) were approximately the same between groups (intervention group $83.8 \%$ vs. nonintervention group $83.3 \%, P=0.726)$. The percentage of patients who received an eye examination during the 12-month period was the lowest of all measures, with $51.2 \%$ of intervention patients having documentation of an eye exam during the period, compared with $48.0 \%$ of nonintervention patients ( $P=0.114$ between groups).

Younger patients received all 5 of the recommended care processes less frequently than older patients $(30.5 \%, 38.0 \%$, and $47.0 \%$ for patients aged $18-48$ years, $49-59$ years, and 60 years or older, respectively, $P<0.001)$. Females had all recommended process of care performed more frequently than males ( $40.4 \%$ vs. $38.0 \%, P=0.019$ ), as did patients who were utilizing insulin (rate among patients with insulin use: $44.9 \%$ compared with $39.7 \%$ and $32.5 \%$ for patients using multiple oral therapy and oral monotherapy, respectively, $P<0.001$ ).

Table 3 presents the result of the multivariate logistic regression analysis including all covariates and intervention group status as a predictor of receiving the diabetes-related processes of care prior to adjustment using propensity scores. In this model, the OR for intervention group status adjusted for covariates was 1.06 (95\% CI=0.90-1.25), indicating that intervention patients were not more likely to have the diabetesrelated processes of care performed compared with the nonintervention group.

The likelihood of having the processes of care performed increased with age, as patients aged 49-59 years were 38\% more likely to receive recommended care compared with patients aged 18-48 years $(\mathrm{OR}=1.38,95 \% \mathrm{CI}=1.23-1.53)$, while patients aged 60 years or older were approximately twice as likely to receive recommended care compared with the youngest age group $(\mathrm{OR}=1.97,95 \% \mathrm{CI}=1.75-2.21)$. Users of multiple oral antidiabetic therapies were 23\% more likely to have the processes of care performed compared with users of oral antidiabetic monotherapy $(\mathrm{OR}=1.23,95 \%$ $\mathrm{CI}=1.11-1.36)$, and patients who utilized insulin at any time during the measurement period were 59\% more likely than users of oral monotherapy to receive these processes of care 


\section{Evaluation of a Program to Improve Diabetes Care Through Intensified Care Management Activities and Diabetes Medication Copayment Reduction}

\section{TABLE 4 Propensity-Score Adjusted Odds Ratio for Receiving All Diabetes- Related Processes of Care for Program Participants (Intervention) Relative to Other Plan Members with Diabetes (Nonintervention) ${ }^{\mathrm{a}}$}

\begin{tabular}{|c|c|c|c|}
\hline & $\begin{array}{c}\text { Beta } \\
\text { Coefficient }\end{array}$ & $\begin{array}{l}\text { Standard } \\
\text { Error }\end{array}$ & $\begin{array}{l}\text { Odds Ratio } \\
\text { (95\% CI) }\end{array}$ \\
\hline \multicolumn{4}{|l|}{ Group } \\
\hline Nonintervention $(n=9,049)$ & - & - & Reference \\
\hline Intervention $(\mathrm{n}=649)$ & 0.0895 & 0.0293 & $\begin{array}{c}1.094 \\
(1.033-1.158) \\
\end{array}$ \\
\hline \multicolumn{4}{|c|}{$\begin{array}{l}\text { avariables represented in the propensity score include age category, gender, } \\
\text { asthma, COPD, the number of unique medications used, and the type of diabetes } \\
\text { medication regimen utilized (oral monotherapy, multiple oral therapy, or any } \\
\text { insulin use). C-statistic }=0.61 \text {. } \\
C I=\text { confidence interval; } C O P D=\text { chronic obstructive pulmonary disease. }\end{array}$} \\
\hline
\end{tabular}

$(\mathrm{OR}=1.59,95 \% \mathrm{CI}=1.41-1.78)$. The likelihood of having the processes of care performed increased with each category of the number of unique medications used during the period, with patients utilizing 21 or more unique medications being most likely to receive the processes of care compared with patients utilizing 5 or fewer medications during the period $(\mathrm{OR}=2.01,95 \% \mathrm{CI}=1.68-2.42)$. Neither gender nor comorbidity status was associated with differences in the likelihood of receiving the processes of care.

The multivariate model incorporating the propensity-score weights included the intervention status variable and the predictors determined to be statistically significant in constructing the propensity-score weighted model. These included age category, gender, the type of diabetes medication utilized, the comorbidities of asthma and COPD, and the number of unique medications used during the period. The propensity scoreadjusted model (Table 4) indicated that intervention group status was associated with a small yet statistically significant increase in the likelihood of receiving all of the recommended care processes during the 12-month time frame (adjusted $\mathrm{OR}=1.09,95 \% \mathrm{CI}=1.03-1.16)$.

We calculated mean per-patient all-cause health care costs accrued during the 12-month study period, categorized as medical care cost; prescription drug cost (for all drugs, not just diabetes drugs); and total costs, which represented the sum of medical and prescription drug costs (Figure 2). Prescription drug costs incurred by the payer over the 12-month period were greater for the intervention group than for nonparticipating patients $(\$ 3,139$ vs. $\$ 2,854$, respectively, $P<0.001)$. The average per-patient pharmacy copayment amounts for all drugs during the measurement period were $\$ 542$ for intervention patients and $\$ 545$ for nonintervention patients $(P=0.341)$. The average per-patient 12 -month all-cause medical care costs were lower for intervention patients; however, this difference in cost
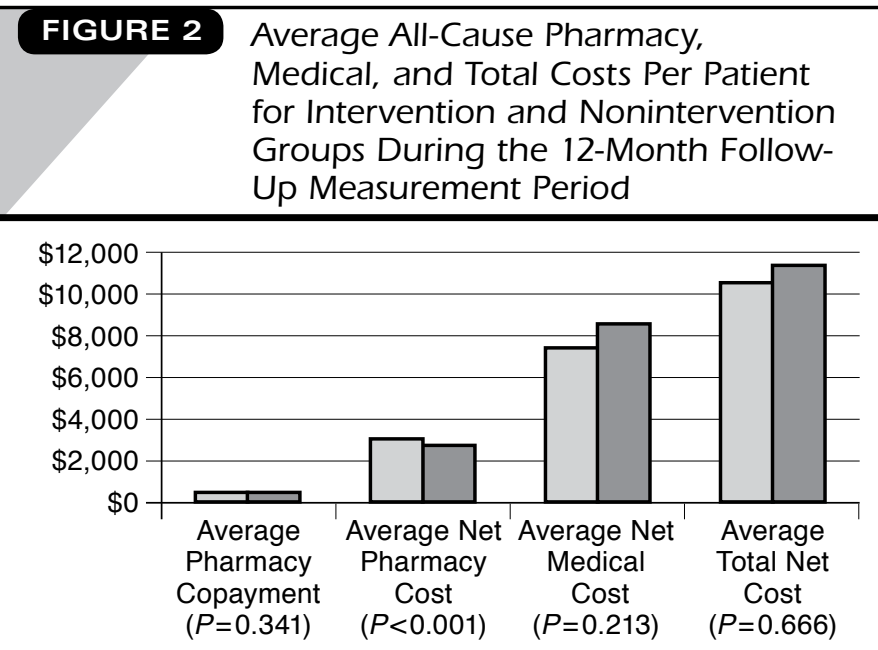

\begin{tabular}{|c|c|c|c|c|c|}
\hline & \multicolumn{2}{|c|}{$\square$ Intervention } & \multicolumn{2}{|c|}{$\square$ Nonintervention } & \multirow[b]{2}{*}{$\begin{array}{c}\text { Total } \\
\text { Pharmacy } \\
\text { and } \\
\text { Medical } \\
\text { Net Plan } \\
\text { Cost }(\$)^{\mathrm{a}}\end{array}$} \\
\hline & $\begin{array}{c}\text { Allowed } \\
\text { Pharmacy } \\
\text { Charge }(\$)\end{array}$ & $\begin{array}{c}\text { Pharmacy } \\
\text { Copayment } \\
(\$)\end{array}$ & $\begin{array}{c}\text { Pharmacy } \\
\text { Net Plan } \\
\text { Cost }(\$)^{a}\end{array}$ & $\begin{array}{l}\text { Medical } \\
\text { Net Plan } \\
\text { Cost }(\$)^{\mathrm{a}} \\
\end{array}$ & \\
\hline $\begin{array}{l}\text { Mean [SD] } \\
\text { inter- } \\
\text { vention }\end{array}$ & $\begin{array}{c}3,681 \\
{[3,755]}\end{array}$ & $\begin{array}{c}542 \\
{[554]}\end{array}$ & $\begin{array}{c}3,139 \\
{[3,426]}\end{array}$ & $\begin{array}{c}7,475 \\
{[17,601]}\end{array}$ & $\begin{array}{c}10,613 \\
{[18,590]}\end{array}$ \\
\hline $\begin{array}{l}\text { Mean [SD] } \\
\text { noninter- } \\
\text { vention }\end{array}$ & $\begin{array}{c}3,399 \\
{[3,802]}\end{array}$ & $\begin{array}{l}545 \\
{[457]}\end{array}$ & $\begin{array}{c}2,854 \\
{[3,938]}\end{array}$ & $\begin{array}{c}8,577 \\
{[22,972]}\end{array}$ & $\begin{array}{c}11,430 \\
{[24,060]}\end{array}$ \\
\hline
\end{tabular}

${ }^{a}$ Net plan cost after subtraction of patient cost share.

$S D=$ standard deviation.

was not statistically significant ( $\$ 7,475$ vs. $\$ 8,577$, respectively, $P=0.213)$. Differences in mean per-patient total costs incurred between the intervention and nonintervention groups were also not statistically significant $(\$ 10,614$ vs. $\$ 11,431$, respectively, $P=0.666$ ).

\section{Discussion}

This study evaluated the results of a multifaceted approach implemented by a commercial health insurer designed to enhance diabetes management by providing patients with intensified oversight and counseling as provided by nurse case managers, coupled with reduced copayments for diabetesrelated medications as an incentive for participating in the intervention including care coordination and agreeing to receive recommended tests. The evaluation of this program focused upon determining rates of performance of 5 recommended care processes and calculating costs of medical care and prescription drugs used during a 12-month period.

As we were interested in evaluating the program during its initial phase, our approach was to compare results among 


\section{Evaluation of a Program to Improve Diabetes Care Through Intensified Care Management Activities and Diabetes Medication Copayment Reduction}

intervention patients with usual care, by forming a comparison group comprising plan members with diabetes who did not participate in the intervention. This approach introduced the possibility of selection bias, as those agreeing to participate in the program may have differed in important ways from nonparticipating members. For example, patients volunteering to participate in the program may have been more inclined than nonvolunteering patients to comply with blood testing for cholesterol monitoring and instructions for glycemic control. While the intervention patients were similar to patients in the comparison group with respect to age distribution and gender, they also utilized insulin more frequently and had a lesser prevalence of COPD (1.1\% vs. $2.7 \%$, respectively, $P=0.017)$ and CAD (11.9\% vs. $16.5 \%, P=0.002)$. These differences suggest that the intervention group in general had a lesser comorbidity burden; yet, they may have had greater diabetes severity, as indicated by the larger percentage of patients with insulin dependence among intervention patients compared with nonintervention patients ( $31.0 \%$ vs. $25.8 \%$, respectively, $P=0.004$ ).

We attempted to control for the possibility of selection bias through the use of a propensity score-weighted model, as derived from a separate multivariate model that determined significant predictors of intervention group status. The results of this model were consistent with the findings of the bivariate analyses, which found that intervention group members received these processes of care at a rate similar to that of patients who did not participate in the intervention $(40.1 \%$ vs. $38.9 \%$, respectively). In the multivariate logistic regression model including all covariates, intervention group status was not predictive of higher rates of performance of the recommended care processes. When assessed using a multivariate model adjusted by propensity score, we found that members enrolled in the program were slightly more likely to have all of the recommended processes of care performed during the measurement period compared with members not enrolled in the program $(\mathrm{OR}=1.09,95 \% \mathrm{CI}=1.03-1.16)$.

While this model indicated a small increase in the likelihood of receiving all tests among intervention members, this finding was likely driven by the between-group difference in the rate of one particular test-eye examination rates $(51.2 \%$ intervention vs. $48.0 \%$ nonintervention, $P=0.114$ ) —as rates of LDL-C and HDL-C testing were slightly lower among intervention patients. In sum, our results indicate that no meaningful difference existed between the intervention and nonintervention groups with regard to performance of the processes of care measured.

Our methodological approach did not permit randomization of patients to the intervention; thus, our findings may have been influenced by between-group differences in the burden of disease and associated intensity of clinical management. Our analyses revealed that the intervention group patients were healthier than nonintervention patients during the study period, as measured by the prevalence of comorbidities and the number of medications utilized. This difference may have yielded closer oversight, more frequent provider contact, and perhaps more aggressive monitoring for nonintervention patients than for the relatively healthier patients in the intervention group. Another proxy for disease burden is the number of unique medications utilized during the study time frame. The patients in the intervention group were less frequently categorized as using more than 10 medications, a finding that further suggests that intervention members were healthier and perhaps less intensely followed by care providers.

Our analyses identified several factors that were associated with increased rates of performance of recommended care. Age was a most significant predictor, as patients aged 60 years or older were nearly twice as likely as patients aged 18 to 48 years to receive these processes of care. Users of antidiabetic monotherapy and patients utilizing 5 or fewer different medications during the period were also less likely to have all of the processes of care performed. While the margin of improvement for the rates of performance of these processes care was greatest among younger patients and patients utilizing antidiabetic monotherapy (compared with users of combination therapies or insulin), further research is necessary to determine how these subgroups specifically may respond to diabetes disease management programs.

For comparison, we contrasted the rates observed in the present study with the NCQA HEDIS benchmarks described earlier. ${ }^{11}$ While we also included HDL-C monitoring in our evaluation, this measure is not included within HEDIS, yet is recommended by the American Diabetes Association. We assumed that HEDIS rates for HDL-C screening would be similar to HEDIS rates for LDL-C screening because both procedures are usually obtained with the same test. In contrasting our intervention sample's rates with those published by the NCQA representing commercial insurers overall, we found intervention patients to have slightly higher rates for yearly Alc testing ( $92.3 \%$ vs. $89 \%$ for HEDIS), LDL-C testing (86.7\% vs. $84.8 \%$ for HEDIS), and medical attention for nephropathy (83.8\% vs. $82.4 \%$ for HEDIS). The percentage of members having a yearly eye examination was slightly lower in our intervention sample, with a rate of $51.2 \%$ versus $56.5 \%$ for HEDIS. Among nonintervention patients in our sample, rates were similar to those of intervention patients and, in fact, higher for lipid measurements. Thus, performance rates for these diabetes-related processes of care among both intervention and nonintervention patients were high, reflecting previous and/ or existing standard disease management programs provided to all members having diabetes. As such, the opportunity for improvement resulting from this diabetes incentive program may have been limited given the high baseline rates for most of these measures.

An additional aim of this study was to determine whether 


\section{Evaluation of a Program to Improve Diabetes Care Through Intensified Care Management Activities and Diabetes Medication Copayment Reduction}

the diabetes incentive program was associated with differences in the costs of medical care and prescription drug utilization. While pharmacy costs were greater among the intervention group members, neither annual per-member medical costs nor total costs differed in statistical significance. The difference in average per-patient overall medical care cost during the period was $\$ 1,102$, with the intervention patients incurring less spending. This finding may reflect the difference in comorbidity prevalence between the intervention and nonintervention groups yet also was influenced by outlier patients having extremely higher costs, as indicated by the difference in the range of costs between groups (intervention patients $\$ 43.9$ to $\$ 199,694, \mathrm{SD}=\$ 17,601$; nonintervention patients $\$ 0$ to $\$ 514,394, \mathrm{SD}=\$ 22,972)$. The outlier values would not dramatically influence the nonparametric statistical test applied, which explains the lack of statistical significance for the difference in medical care cost experienced between the groups. Further evaluation of this program over time will be informative in determining if cost reduction among intervention participants is achieved over the longer term.

Intervention participants incurred higher expenditures for prescription drug utilization compared with nonintervention patients. This finding aligned with program expectations, given the expected increased cost borne by the plan for the reduced copayments for antidiabetic medications among intervention members. Yet further analyses revealed that dispensings for lower-cost generic medications were more frequent among nonintervention members. The mean (SD) generic dispensing ratio for all drugs (not just diabetes drugs) was 62.1\% (22.4\%) among intervention patients compared with 65.4\% (23.0\%) among nonintervention patients $(P<0.001$, t-test, 2 sided). Due to the limitations of our observational study design, we were unable to determine the frequency of dispensing of generic medications among those patients who were offered the program but declined to participate, which would enable a better understanding of the relative value of copayment reduction as perceived by patients using brand-name medication. Further research examining the effects of copayment reduction as a component of disease management in diabetes is warranted, particularly considering that the value of medication therapies is likely to be an increasingly important aspect of formulary designs.

\section{Limitations}

Several limitations of this study should be recognized. First and most importantly, we were unable to make comparisons with prior periods to enable a pre-intervention versus postintervention analysis. The intervention may have provided greater gains in performance rates or greater reductions in cost from the previous year for those participating in the program, yet we were unable to measure this possible effect. Also, given the brief 12 -month measurement time frame, we were unable to determine the temporal relationships between variables. For example, a member could have been diagnosed with asthma on the last day of the 12-month period and would have been classified as having asthma for the entire study period.

Second, we evaluated all-cause costs but did not examine diabetes-related costs specifically. We sought to determine the overall cost impact associated with this disease management program, which included components potentially affecting expenditures for both diabetes and other health conditions, such as cardiovascular and renal disease. Additionally, we believed that reductions in diabetes-related costs would be more likely than reductions in all-cause costs to occur after the end of the 12-month measurement period. Nevertheless, there may have been a significant impact of this disease management program on diabetes-related costs in particular that was not identified.

Third, we did not evaluate patient adherence to antidiabetic medications. While it was envisioned that copayment reduction might promote medication adherence, the copayment incentive was primarily designed to encourage patient interest and involvement in the disease management program to enhance the quality of care overall, as assessed according to the diabetes-related care processes evaluated in this study. To participate in the program and qualify for the copayment reduction, patients agreed to have an annual physical examination, have Alc and cholesterol testing performed at recommended intervals, and work with a care coordinator. Patients were not required to achieve a particular level of medication adherence. Thus, we focused our evaluation on the program components described in the patient pledge.

Fourth, the all-or-none approach to the composite measure, which represented whether all of the processes of care were performed, could have missed overall better care. For example, receipt of 4 of 5 measures and receipt of 0 of 5 measures were both classified as failing to receive all recommended care. However, it was evident from the rates observed for each specific measure that the processes of care were performed with similar frequency for both the intervention and nonintervention members. Another important limitation pertains to the lack of data available to identify the achievement of therapeutic goals. We determined only whether a test was performed; we were unable to determine the percentage of members who had their blood glucose and lipid levels reduced to recommended goals. Patients could have had all tests performed but still have values representing high risk of diabetic complications.

A fifth limitation of this study is the nature of the administrative data source, which contained information about paid claims only. Any diagnosis, procedure, or medication dispensing that occurred and was not recorded or that was paid out-of-pocket was not included in this study. Additionally, we assumed that dispensed medications were actually taken as prescribed by members, and in this analysis we did not assess adherence to dispensed medications. 


\section{Evaluation of a Program to Improve Diabetes Care Through Intensified Care Management Activities and Diabetes Medication Copayment Reduction}

Sixth, members choosing to participate in this diabetes incentive program could have been more motivated in managing their health and thus utilized more health care resources. As with any observational study, the lack of randomization allows for the presence, and influence, of biases that we have not identified. Seventh, we chose to include only patients who were utilizing antidiabetic medications. Our results may have differed if we also included patients who were identified as having diabetes but not yet utilizing pharmacotherapy for the condition.

\section{Conclusions}

As measured by a composite indicator comprising 5 diabetesrelated processes of care, receipt of recommended care was not significantly different for patients participating in a disease management program that included copayment reduction for antidiabetic medications and intensive health coaching, compared with nonparticipating patients with diabetes. Younger patients and those utilizing oral antidiabetic monotherapy were less likely to have these recommended processes of care performed. While prescription drug expenditures incurred by the plan were greater for intervention patients, between-group differences in total costs for medications and medical care were not statistically significant. Further follow-up is required to determine the success of this program over the longer term in promoting quality of care and achieving cost reductions and improved health outcomes.

\section{Authors}

STEPHEN J. KOGUT, PhD, MBA, is Associate Professor; and BRIAN QUILLIAM, PhD, is Associate Professor, College of Pharmacy, University of Rhode Island, Kingston, Rhode Island. SCOTT JOHNSON, PharmD, MS, is Staff Pharmacist, Peninsula Regional Medical Center, Salisbury, Maryland. TARA HIGGINS, BS Pharm, CDOE, is Clinical Pharmacist, Blue Cross \& Blue Shield of Rhode Island, Providence, Rhode Island.

AUTHOR CORRESPONDENCE: Stephen J. Kogut, PhD, MBA, College of Pharmacy, University of Rhode Island, 41 Lower College Rd., Kingston, RI 02881. Tel.: 401.874.5370; E-mail: Kogut@URI.edu.

\section{DISCLOSURES}

This work was in part supported by an educational grant from Takeda Pharmaceuticals provided to the University of Rhode Island College of Pharmacy towards a graduate fellowship program involving research collaboration with Blue Cross \& Blue Shield of Rhode Island.

Concept and design were performed by Higgins, Johnson, and Kogut. Data were collected primarily by Higgins and were interpreted primarily by Johnson, Quilliam, and Kogut. The manuscript was written primarily by Johnson and Kogut and was revised primarily by Kogut with Johnson's assistance.

\section{ACKNOWLEDGEMENTS}

The authors acknowledge the work of Aisling Caffrey, MS, PhD, in assisting with measure definitions and specifications.

\section{REFERENCES}

1. Centers for Disease Control and Prevention. National diabetes fact sheet: national estimates and general information on diabetes and prediabetes in the United States, 2011. Atlanta, GA: U.S. Department of Health and Human Services, Centers for Disease Control and Prevention, 2011. Available at: http://www.cdc.gov/diabetes/pubs/pdf/ndfs_2011.pdf. Accessed April 2, 2012.

2. Narayan KM, Boyle JP, Thompson TJ, Sorensen SW, Williamson DF. Lifetime risk for diabetes mellitus in the United States. JAMA. 2003;290(14):1884-90. Available at: http://jama.ama-assn.org/content/290/14/1884.full.pdf+html. Accessed April 2, 2012.

3. Gambert SR, Pinkstaff S. Emerging epidemic: diabetes in older adultsdemography, economic impact, and pathophysiology. Diabetes Spectrum. 2006;19(4):221-28. Available at: http://spectrum.diabetesjournals.org/content/19/4/221.full.pdf+html. Accessed April 2, 2012.

4. Hogan P, Dall T, Nikolov P; American Diabetes Association. Economic costs of diabetes in the U.S. in 2002. Diabetes Care. 2003;26(3):917-32. Available at: http://care.diabetesjournals.org/content/26/3/917.full.pdf + html. Accessed April 2, 2012

5. Cowie CC, Rust KF, Ford ES, et al. Full accounting of diabetes and prediabetes in the U.S. population in 1988-1994 and 2005-2006. Diabetes Care. 2009;32(2):287-94. Available at: http://care.diabetesjournals.org/content/32/2/287. Accessed April 2, 2012

6. American Diabetes Association. Economic costs of diabetes in the U.S. in 2007. Diabetes Care. 2008;31(3):596-615. Available at: http://care.diabetesjournals.org/content/31/3/596.abstract. Accessed April 2, 2012.

7. American Diabetes Association. Standards of medical care in diabetes-2010. Diabetes Care. 2010;33(Suppl 1):S4-S10. Available at: http://care. diabetesjournals.org/content/33/Supplement_1/S11.full.pdf+html. Accessed April 2, 2012. 


\section{Evaluation of a Program to Improve Diabetes Care Through Intensified Care Management Activities and Diabetes Medication Copayment Reduction}

8. Turner RC, Millns H, Neil HA, et al. Risk factors for coronary artery disease in non-insulin dependent diabetes mellitus: United Kingdom Prospective Diabetes Study (UKPDS:23). BMJ. 1998;316(7134):823-28. Available at: http://www.ncbi.nlm.nih.gov/pmc/articles/PMC28484. Accessed April 2, 2012.

9. Koro CE, Lee BH, Bowlin SJ. Antidiabetic medication use and prevalence of chronic kidney disease among patients with type 2 diabetes mellitus in the United States. Clin Ther. 2009;31(11):2608-17.

10. Zhang X, Saaddine JB, Chou CF, et al. Prevalence of diabetic retinopathy in the United States, 2005-2008. JAMA. 2010;304(6):649-56. Available at: http://jama.ama-assn.org/content/304/6/649.long. Accessed April 2, 2012.

11. National Committee for Quality Assurance. The state of health care quality: value, variation and vulnerable populations. 2009. Available at: http:// www.ncqa.org/Portals/0/Newsroom/SOHC/SOHC_2009.pdf. Accessed April 2, 2012

12. U.S. Department of Health and Human Services. Agency for Healthcare Research and Quality. National healthcare quality report, 2010. AHRQ Publication No. 11-0004. March 2011. Available at: http://www.ahrq.gov/ qual/nhqr10/nhqr10.pdf. Accessed April 2, 2012.

13. McCall N, Cromwell J. Results of the Medicare Health Support diseasemanagement pilot program. N Engl J Med. 2011;365(18):1704-12. Available at: http://www.nejm.org/doi/full/10.1056/NEJMsal011785. Accessed April 2,2012

14. Sönnichsen AC, Winkler H, Flamm M, et al. The effectiveness of the Austrian disease management programme for type 2 diabetes: a clusterrandomised controlled trial. BMC Fam Pract. 2010;11:86. Available at: http:// www.ncbi.nlm.nih.gov/pmc/articles/PMC2989935. Accessed April 2, 2012.

15. Hogg W, Lemelin J, Dahrouge S, et al. Randomized controlled trial of anticipatory and preventive multidisciplinary team care: for complex patients in a community-based primary care setting. Can Fam Physician. 2009;55(12):e76-85. Available at: http://www.ncbi.nlm.nih.gov/pmc/articles/ PMC2793206. Accessed April 2, 2012.

16. Cranor CW, Bunting BA, Christensen DB. The Asheville Project: longterm clinical and economic outcomes of a community pharmacy diabetes care program. J Am Pharm Assoc (Wash). 2003;43(2):173-84.
17. Fera T, Bluml BM, Ellis WM. Diabetes Ten City Challenge: Final economic and clinical results. J Am Pharm Assoc (2003). 2009;49(3):383-91.

18. Garrett DG, Martin LA. The Asheville Project: participants' perceptions of factors contributing to the success of a patient self-management diabetes program. J Am Pharm Assoc (Wash). 2003;43(2):185-90.

19. Neumann PJ. Evidence-based and value-based formulary guidelines. Health Aff (Millwood). 2004;23(1):124-34. Available at: http://content. healthaffairs.org/content/23/1/124.long. Accessed April 2, 2012.

20. Fairman KA, Curtiss FR. What do we really know about VBID? Quality of the evidence and ethical considerations for health plan sponsors. J Manag Care Pharm. 2011;17(2):156-74. Available at: http://www.amcp.org/data/ jmcp/156-174.pdf.

21. Robinson JC. Comparative effectiveness research: from clinical information to economic incentives. Health Aff (Millwood). 2010;29(10):1788-95.

22. Gibson TB, Mahoney J, Ranghell K, Cherney BJ, McElwee N. Valuebased insurance plus disease management increased medication use and produced savings. Health Aff (Millwood). 2011;30(1):100-08.

23. Chernew ME, Shah MR, Wegh A, et al. Impact of decreasing copayments on medication adherence within a disease management environment. Health Aff (Millwood). 2008;27(1):103-12. Available at: http://content.healthaffairs. org/content/27/1/103.long. Accessed April 2, 2012.

24. Chang A, Liberman JN, Coulen C, Berger JE, Brennan TA. Value-based insurance design and antidiabetic medication adherence. Am J Pharm Benefits. 2010;2(1):39-34. Available at: http://www.ajpblive.com/media/pdf/ Chang_2-1.pdf. Accessed April 2, 2012.

25. National Committee for Quality Assurance (NCQA). Comprehensive Diabetes Care. HEDIS 2009, Vol. 2, Technical Specifications. 2009;(2):13448. Available at: http://www.ncqa.org/tabid/784/Default.aspx. Accessed April 2, 2012.

26. D'Agostino RB Jr. Propensity score methods for bias reduction in the comparison of a treatment to a non-randomized control group. Stat Med. 1998;17(19):2265-81. 


\section{Evaluation of a Program to Improve Diabetes Care Through Intensified Care Management Activities and Diabetes Medication Copayment Reduction}

\section{APPENDIX ICD-9-CM and CPT Codes Applied in Determining Process-of-Care Rates and Comorbidities ${ }^{\mathrm{a}}$}

Alc (CPT)

83036,83037

Asthma (ICD-9-CM)

493.XX

COPD (ICD-9-CM)

491.XX, 492.XX, 496

Coronary artery disease (ICD-9-CM)

410.XX - 414.XX

Diabetes mellitus (ICD-9-CM)

250.XX, 357.2, 362.0X, 366.41, 648.0X

Eye examination ${ }^{\mathrm{b}}(\mathrm{CPT} / \mathrm{ICD}-9-\mathrm{CM})$

CPT: 67028, 67030, 67031, 67036, 67038-67043, 67101, 67105, 67107, 67108, 67110, 67112, 67113, 67121, 67141, 67145, 67208, 67210, 67218, 67220, $67221,67227,67228,92002,92004,92012,92014,92018,92019,92225,92226,92230,92235,92240,92250,92260$

ICD-09-CM: 14.1-14.5, 14.9, 95.02-95.04, 95.11, 95.12, 95.16

HDL-C (CPT)c

$80061,83700,83701,83704,83718$

Heart failure (ICD-9-CM)

398.91, 402.01, 402.11, 402.91, 404.01, 404.11, 404.91, 428.XX

LDL-C (CPT)

$80061,83700,83701,83704,83721$

Medical attention for nephropathyd (CPT/ICD-9-CM)

81000-81003, 81005, 82042, 82043, 82044, 84156, 36145, 36800, 36810, 36815, 36818, 36819-36821, 36831-36833, 50300, 50320, 50340, 50360, 50365, 50370, 50380, 90920, 90921, 90924, 90925, 90935, 90937, 90939, 90940, 90945, 90947, 90989, 90993, 90997, 90999,99512

ICD-9-CM: 250.4, 403, 404, 405.01, 405.11, 405.91, 580-588, 753.0, 753.1, 791.0, V42.0, V45.1, V56, 38.95, 39.27, 39.42, 39.43, 39.53, 39.93-39.95, $54.98,55.4-55.6$

Mental health diagnoses (ICD-9-CM)e

293.XX, 294.XX, 295.XX, 296.XX, 297.XX, 298.XX, 299.XX

Polycystic ovary syndrome (ICD-9-CM)

256.4

aCode sets based upon HEDIS specifications except where noted. 20

${ }^{b} \mathrm{CPT}$ code list also includes codes for eye procedures as a proxy for dilated eye exam.

cHDL-C testing is not a HEDIS measure.

${ }^{d}$ Codes include a range of procedures that indicate provider attention to renal function per HEDIS specifications.

eIncludes code sets for psychosis, schizophrenia, depression, and paranoia.

$A 1 c=$ hemoglobin $A 1 c ; C O P D=$ chronic obstructive pulmonary disease; $C P T=$ Current Procedural Terminology; HDL- $C=$ high-density lipoprotein cholesterol;

HEDIS = Healthcare Effectiveness Data and Information Set; ICD-9-CM = International Classification of Diseases, Ninth Revision, Clinical Modification; LDL-C=lowdensity lipoprotein cholesterol. 